\title{
The urgent need for broad-spectrum antiviral drugs
}

\author{
Volume 2 Issue 2 - 2015 \\ Jocelyn Yelle
Antiviral Inteli Strat Inc., Canada
}

\section{Editorial}

During the last decades, human populations have been exposed to several new viral pathogens such as Ebola and Marburg viruses, Lassa fever virus, SARS- and MERS- Corona viruses, Hantaviruses, Kyasanur Forest virus and others, often with disastrous consequences. ${ }^{1-6}$ While in several cases these represent sporadic outbreaks that wane over a period of a few weeks, the events of 2014 have exposed our vulnerability to such highly pathogenic agents with the unprecedented Ebola virus epidemic which is barely under control after more than a year in West Africa. ${ }^{7}$ These outbreaks tend to occur in developing countries; however they can rapidly expand and reach other parts of the globe through basic transportation facilities. The 2014 Ebola virus outbreak has provided a particularly interesting opportunity to test our ability to react and respond to a major health crisis in one of the worst possible situation. Sadly, the results have exposed several weaknesses and underlined the necessity to review our entire approach to these natural calamities. Besides the lack of basic facilities and limited infrastructure in the affected countries, the absence of an adequate vaccine to protect local populations and healthcare personal, and of approved antiviral drugs to treat infected patients, resulted in a true humanitarian disaster with thousands of deaths.

Two traditional approaches, vaccines and antiviral drugs, represent our main weapons to control viral outbreaks. Vaccines are designed to elicit a highly specific immune response to a pathogen. As a consequence, a newly found virus must be at least partially characterized in order to prepare an effective vaccine against it. In addition, the vaccine design and evaluation process together can take a significant amount of time. In the particular case of Ebola virus, despite the fact that there were already existing vaccine candidates against the virus that were rushed into clinical evaluation under what is probably the fastest testing scheme ever seen, no vaccine has yet completed phase 2 efficacy assessment more than a year after the first infected patients were reported. Clearly, in the case of an outbreak by a new viral pathogen, de novo vaccine preparation alone does not represent the best approach to avoid a health crisis and prevent human casualties.

Specificity represents also an important element in the design of traditional antiviral molecules that limits potential toxicity in the host. For instance, HIV protease inhibitors on the market for HIV/AIDS treatment have an efficiency concentration against the viral protease that is several hundred times lower than the concentration needed to inhibit cellular aspartic proteases. ${ }^{8}$ Likewise, marketed inhibitors of viral polymerases display a high level of specificity vis-à-vis their viral target and interfere only minimally with cell enzymes. This high level of specificity can only be achieved when a detailed knowledge of the viral target's structure and function is available, which is not the case when a new pathogen first emerges in human populations. A few compounds showing activity against the Ebola virus have been reported in the literature over the years, but none of them had been submitted to significant clinical evaluation on the ground in affected countries before the 2014 outbreak. ${ }^{7}$ At this point in time, their real efficacy in infected patients has not been reported. Since one can

\author{
Correspondence: Jocelyn Yelle, Antiviral InteliStrat Inc, 3077 \\ Edouard Montpetit Street, Suite 406, Laval, Quebec, H7T 2K8, \\ Canada, Tel I-5|4-8|6-8856, Fax |-5|4-227-53|5, \\ Email yellej@videotron.ca, jyelle@antiviralintelistrat.com
}

Received: January 23, 2015 | Published: January 25, 2015

expect that other viral outbreaks will certainly affect us in the near future, a new plan is needed to prevent tragedies such as the 2014 Ebola epidemic. Based on the experience and knowledge acquired through several viral outbreaks in the past, an interesting novel strategy deserves attention, an approach that is immediately applicable to the field of antiviral drugs. Instead of focusing on highest possible specificity, it might be a good idea to take one step back and to look for compounds that show activity against a broader range of viruses, e.g. molecules that are able to inhibit targets present in more than one virus species.

Viruses harbor common well-defined targets that often show a high level of conservation at the genomic and/or structural level, at least within viral families. These targets, especially protease and polymerase, represent well-known types of protein and standard targets for the Pharma industry. Several viral proteases belong to the same protease subfamily, which means that there must exist some similarities at the active site of these enzymes, which could allow some inhibitor molecules to be active against more than one of these enzyme entities, potentially across viral families. Even more interesting, several of the most lethal viral pathogens belong to viral families harboring an RNA genome (more often in general than DNA viruses), and thus encode an RNA polymerase to synthesize new copies of the viral RNA. Again, these enzymes do have structural similarities at the level of their catalytic site that can be exploited to identify or design broad-spectrum inhibitors. Arguably, RNA polymerases could be better suited for this approach than proteases. Compounds synthesized over the years as inhibitors for these enzyme targets should be systematically screened against a panel of proteases and polymerases from various families of viruses, to identify those molecules with activity against multiple viral enzymes. The enzymes should be chosen among families of viruses that represent major pathogens in terms of pathogenicity and mortality rates in humans such as Arena viruses, Bunya viruses, Corona viruses, Filo viruses, Toga viruses, etc. The compounds should be tested in parallel against a panel of enzymes from these viruses, which would be continually updated as new viral pathogens are identified.

Besides enzymatic assays, the most promising compounds should be evaluated in cell culture assays and whenever possible, in animal models. In parallel, basic toxicology assays should be performed to keep only compounds with the best safety profile. While the desired 
compounds are expected to inhibit viral replication with significant potency, they would likely display reduced specificity compared to other drugs so their safety profile will need to be closely monitored. On the other hand, ideally the selected drugs would be used over a relatively short period of time, eliminating long-term potential toxicity issues. This concept of an arsenal of antiviral drugs with broad-spectrum activity among viruses, ready to be used as a first line of defense in case of emergency when a new lethal virus emerges, is not new and has been proposed by others. ${ }^{9-10}$ However, it has not yet been pursued systematically as a way to respond to viral outbreaks, in part because such compounds are still limited in number or have been discarded in the past for lack of specificity, and also because there was no financial interest from the Pharma industry to explore other ways to address viral outbreaks. With the realization that these pathogens can easily reach populations in industrialized countries and cause havoc, a new perception is taking place.

Some promising broad-spectrum molecules already exist but their full potential has not been explored..$^{9,11-15}$ Ribavirin, perhaps the oldest and most known broad-spectrum antiviral, has been used for years as part of the traditional HCV treatment and in special situations where no other drugs were available. ${ }^{16}$ Its many side effects and variable efficacy make it a less than ideal compound for this strategy. Other compounds with activity against more than one type of virus have been reported in recent years, and some of them have even been tested against the Ebola virus. ${ }^{7}$ Besides enzyme inhibitors, compounds disrupting the viral envelope or blocking viral entry in host cells have also been recently reported and could also have a place in this new paradigm of first line response to lethal viruses. This strategy needs to be put in place quickly. Fortunately, it seems that there is a slow but decisive evolution towards seeking this type of compounds, to be used as first line therapy in cases of outbreaks involving lethal viral strains. Let's hope the lessons learned from last year's Ebola outbreak will lead to a better adapted strategy to face these viral threats.

\section{Conflicts of interest}

The author is President of Antiviral Inteli Strat Inc., He declares having no financial links or interests in other organizations listed in the manuscript.

\section{Acknowledgments}

None.

\section{References}

1. Seah SK. Lassa, Marburg and Ebola: newly described African fevers. Can Med Assoc J. 1978;118(4):347-348, 350.
2. Feldmann H. Truly Emerging - A New Disease Caused by a Novel Virus. N Engl J Med. 2011;364(16):1561-1563.

3. Anderson LJ, Baric RS. Emerging Human Coronaviruses - Disease Potential and Preparedness. N Engl J Med. 2012;367(19):1850-1852.

4. Peiris JS, Lai ST, Poon LL, et al. Coronavirus as a possible cause of severe acute respiratory syndrome. Lancet. 2003;361(9366):1319-1325.

5. Yadav PD, Shete AM, Patil DY, et al. Outbreak of Kyasanur Forest disease in Thirthahalli, Karnataka, India, 2014. Int J Infect Dis. 2014;26:132-134.

6. Kruger DH, Figueiredo LT, Song JW, et al. Hantaviruses-Globally emerging pathogens. J Clin Virol. 2014;S1386-S6532(14):00372-2.

7. Yelle J. The 2014 Ebola Virus Outbreak in West Africa: Current Perspectives for Prevention and Treatment. J Hum Virol Retrovirol. 2014;1(4):00024.

8. Abbenante G, Fairlie DP. Protease inhibitors in the clinic. Med Chem. 2005;1(1):71-104

9. Debing Y, Jochmans D, Neyts J. Intervention strategies for emerging viruses: use of antivirals. Curr Opin Virol. 2013;3(2):217-224.

10. Leyssen P, De Clercq E, Neyts J. Molecular strategies to inhibit the replication of RNA viruses. Antiviral Res. 2008;78(1):9-25.

11. Chan JF, Chan KH, Kao RY, et al. Broad-spectrum antivirals for the emerging Middle East respiratory syndrome coronavirus. J Infect. 2013;67(6):606-616.

12. Florescu DF, Keck MA. Development of CMX001 (Brincidofovir) for the treatment of serious diseases or conditions caused by dsDNA viruses. Expert Rev Anti Infect Ther. 2014;12(10):1171-1178.

13. Aman MJ, Kinch MS, Warfield K, et al. Development of a broadspectrum antiviral with activity against Ebola virus. Antiviral Res. 2009;83(3):245-251.

14. Elshabrawy HA, Fan J, Haddad CS, et al. Identification of a broadspectrum antiviral small molecule against severe acute respiratory syndrome coronavirus and Ebola, Hendra, and Nipah viruses by using a novel high-throughput screening assay. $J$ Virol. 2014;88(8):4353-4365.

15. Safronetz D, Falzarano D, Scott DP, et al. Antiviral efficacy of favipiravir against two prominent etiological agents of hantavirus pulmonary syndrome.. 2013;57(10):4673-4680.

16. Thomas E, Ghany MG, Liang TJ. The application and mechanism of action of ribavirin in therapy of hepatitis C. Antivir Chem Chemother. 2013;23(1):1-12. 